


\title{
Shack-Hartmann wave front measurements in cortical tissue for deconvolution of large three-dimensional mosaic transmitted light brightfield micrographs
}

\author{
M. OBERLAENDER*, P.J. BROSER $\dagger$, B. SAKMANN* \\ \& S. HIPPLER \\ * Max Planck Institute of Neurobiology Research Group 'Cortical Column In Silico', Am Klopferspitz \\ 18, Martinsried 82152, Germany \\ $\dagger$ The Royal London Hospital Whitechapel, Whitechapel Road, London E1 1BB, U.K. \\ $\ddagger$ Max Planck Institute for Astronomy Department of Adaptive Optics, Koenigstuhl 17, Heidelberg \\ 69117, Germany
}

Key words. Aberrations, mosaic TLB microscopy, neurons, refractive index,

Shack-Hartmann.

\begin{abstract}
Summary
We present a novel approach for deconvolution of 3D image stacks of cortical tissue taken by mosaic/opticalsectioning technology, using a transmitted light brightfield microscope. Mosaic/optical-sectioning offers the possibility of imaging large volumes (e.g. from cortical sections) on a millimetre scale at sub-micrometre resolution. However, a blurred contribution from out-of-focus light results in an image quality that usually prohibits 3D quantitative analysis. Such quantitative analysis is only possible after deblurring by deconvolution. The resulting image quality is strongly dependent on how accurate the point spread function used for deconvolution resembles the properties of the imaging system. Since direct measurement of the true point spread function is laborious and modelled point spread functions usually deviate from measured ones, we present a method of optimizing the microscope until it meets almost ideal imaging conditions. These conditions are validated by measuring the aberration function of the microscope and tissue using a Shack-Hartmann sensor. The analysis shows that cortical tissue from rat brains embedded in Mowiol and imaged by an oil-immersion objective can be regarded as having a homogeneous index of refraction. In addition, the amount of spherical aberration that is caused by the optics or the specimen is relatively low. Consequently the image formation is simplified to refraction between the embedding and immersion medium and to 3D diffraction at the finite entrance pupil of the objective. The resulting model point spread function is applied to the image stacks by linear or iterative deconvolution algorithms. For the presented dataset

Correspondence to: Marcel Oberlaender. Tel: +49 (0)89 85783285 ; fax: +49 (0)89 8578 3300; e-mail: oberlaender@neuro.mpg.de
\end{abstract}

of large 3D images the linear approach proves to be superior. The linear deconvolution yields a significant improvement in signal-to-noise ratio and resolution. This novel approach allows a quantitative analysis of the cortical image stacks such as the reconstruction of biocytin-stained neuronal dendrites and axons.

\section{Introduction}

Three-dimensional imaging of cortical tissue on a millimetre scale and at sub-micrometre resolution is a challenging task in neuroscience. Detailed tracing of morphology of cortical and thalamic neurons (Oberlaender et al., 2007), as well as the visualization of neuronal networks in the hippocampus (Dodt et al., 2007) are two recent examples.

Most 3D imaging approaches for quantitative analyses are based on fluorescent techniques, like two-photon or confocal microscopy (Schmitt et al., 2004; Wearne et al., 2005). However, the limited field of view, the finite lifetime of the fluorescent dye, bleaching and noise artefacts of the chargecoupled device (CCD) camera (Lee et al., 1998) (Poissonian distributed noise from emitted photons, dark current noise, additive Gaussian distributed readout noise, quantization noise) as well as relatively high costs (Holmes \& O'Connor, 2000) are substantial problems for high-resolution imaging (e.g. $0.116 \times 0.116 \times 0.5 \mu \mathrm{m})$ of large volumes of tissue (e.g. $1.5 \mathrm{~mm} \times 1.5 \mathrm{~mm} \times 100 \mu \mathrm{m})$.

Recently, a transmitted light brightfield (TLB) microscope equipped with mosaic-scanning (Chow et al., 2006) and optical-sectioning (Agard, 1984) was used to overcome these limitations. Throughout this article biocytin-stained neuronal processes (Horikawa \& Armstrong, 1988) in rat cortex 


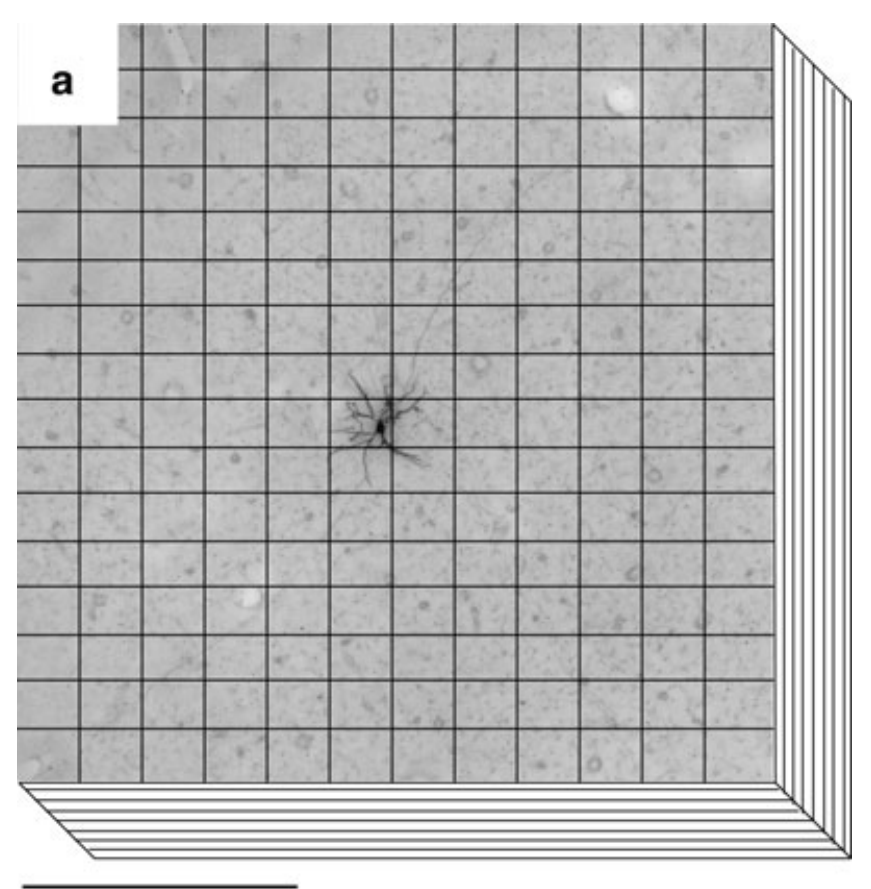

$500 \mu \mathrm{m}$

Fig. 1. Illustration of mosaic/optical-sectioning TLB microscopy for 3D high-resolution imaging of large volumes of cortical tissue (biocytinstained neuronal projections). Each rectangle refers to one field of view of a $40 \times$ objective which yields a lateral sampling of $0.116 \mu \mathrm{m}$ per pixel. Three dimensionality arises by recording a series of focal planes separated by $0.5 \mu \mathrm{m}$.

function as an example of light-absorbing structures (Fig. 1) that can be imaged by such a TLB system.

Despite the fact that the TLB microscope is probably the most widely used instrument in a lifescience laboratory (Holmes \& O'Connor, 2000), it has one significant disadvantage in comparison to the above-mentioned high-resolution fluorescent techniques. Out-of-focus light caused by diffraction effects due to the finite aperture of the objective and aberrations induced by the optical components or the specimen result in a blurred contribution to each $2 \mathrm{D}$ image plane. As a consequence the resolution, especially along the optical $(z-)$ axis, is not sufficient to obtain a high-resolution visualization and quantification (e.g. of neuronal morphology) from raw TLB data (Holmes \& O'Connor, 2000).

The optical limits of the imaging system can be described in terms of its 3D point-spread function (PSF) or its Fourier transform the optical transfer function (OTF) (Born \& Wolf, 2003). The PSF specifies the appearance of an idealized point [delta function (Bronshtein et al., 2003a)] after being imaged by the microscope system. Neglecting coherency effects and noise caused by the CCD camera, the imaging of an object is then given in terms of a convolution of the PSF $s(x, y, z)$ with the object's intensity distribution $o(x, y, z)$ (Agard, 1984;
Beverage et al., 2002):

$$
i(x, y, z)=o(x, y, z) \otimes s(x, y, z)
$$

where $i(x, y, z)$ refers to the stack of recorded 2D images and the symbol $\otimes$ denotes the convolution operation described in standard textbooks (Bronshtein et al., 2003b).

According to Eq. (1) image formation could be reverted if the PSF is known. This procedure is called deconvolution or image restoration. The intensity distribution of the object could then be obtained, apart from spatial frequency domains of the OTF that are dominated by noise or at the zero crossing (Chomik et al., 1997). For any further considerations the noise caused by the CCD camera, which becomes more dominant with low light intensities (Lee et al., 1998) will be neglected. The camera's exposure time is automatically adjusted to guarantee that stained neuronal processes cover a maximum grey-value range and to ensure none-zero background of unstained tissue (Oberlaender et al., 2007). Therefore, throughout this paper, noise refers to unstained lightabsorbing cortical tissue that surrounds stained neurons and does not include the CCD noise which can be problematic in fluorescence microscopy.

It is obvious that the deconvolution result approaches its optimal performance if the applied PSF resembles the properties of the imaging system. Hence, a correct PSF will reduce artefacts from unstained background that are interpreted as noise and consequently improves the quality of an incoherently illuminated wide-field TLB image stack in terms of signal-to-noise ratio (SNR), where SNR refers to the ratio between stained neuronal processes and the unstained tissue background. In addition the resolution will improve significantly, making a high-resolution visualization and quantification of wide-field data possible. In consequence, the correct determination of the microscope's PSF is the essential challenge in TLB microscopy.

Several theoretical (Frieden, 1967; Gibson \& Lanni, 1991; Philip, 1999) and experimental (Agard et al., 1989; Hiraoka et al., 1990) methods have been described to obtain the 3D PSF of a microscope. A common approach is to measure the PSF by recording a series of $2 \mathrm{D}$ images of a sub-resolution object with different amounts of defocus, where sub-resolution refers to an object whose dimensions are smaller than the diffraction limit (Born \& Wolf, 2003) of the microscope's objective. Fluorescent objects (beads) of this size yield a weak signal that requires an extremely sensitive detector and limits the axial range over which the PSF can be measured (Hiraoka et al., 1990; Vandervoort \& Strasters, 1995; Dusch et al., 2007). When the optical influences of the tissue need to be investigated, this method is exceedingly difficult (Holmes \& O'Connor, 2000) for two reasons. First, the fluorescent bead needs to be inserted into the tissue making its signal even fainter, and second it is laborious to measure the PSF for every single preparation.

The alternative, application of a theoretically modelled PSF for deconvolution, is also delicate. Significant discrepancy 
has been noted between theoretical and experimental 3D PSFs. The difference results in part from inaccurate choice of parameter values (e.g. unknown refractive index of tissue), or the theoretical model assumes ideal imaging conditions, e.g. the absence of any aberration (Beverage et al., 2002).

Since a correct description of the imaging system in terms of its PSF is not trivial and both, theoretical and experimental approaches seem to be not satisfying, alternative solutions have been suggested. The 'blind deconvolution' approach (Holmes \& O'Connor, 2000) uses a nonlinear, iterative and constrained maximum likelihood estimation (MLE) algorithm that requires the PSF not as an input parameter, but reconstructs the PSF concurrently with the image data. However, we argue that this approach is only applicable for rather small image stacks (one or a few fields of view), because application of this filter on large image stacks with high resolution has severe limitations.

First, iterative algorithms are strongly influenced by the estimation of the background intensity (van Kempen \& van Vliet, 2000a). An underestimation of background intensities will make the constraint ineffective. This results in a performance similar to that obtained by linear restoration algorithms. An overestimation of the background is problematic, since it results in clipping of the object intensities. Usually, TLB image stacks of the above described dimensions yield neither a uniform background nor can it be assumed that structures dwell in similar intensity ranges throughout such volumes. These inhomogeneous conditions influence the background estimation negatively and result possibly in a loss of performance. Examples for this phenomenon will be illustrated in the result section.

The second limitation is a rather practical one. An 8-bit image stack of the size of $1.5 \mathrm{~mm} \times 1.5 \mathrm{~mm} \times 100 \mu \mathrm{m}$ with a sampling rate of $0.116 \times 0.116 \times 0.5 \mu \mathrm{m}$ comprises a data volume of approximately $25 \mathrm{~GB}$. Even on a multi-core server computer a MLE deconvolution carried out by Huygens Professional 2.9 deconvolution software (Scientific-VolumeImaging, 1995-2007) takes around $24 \mathrm{~h}$, whereas a linear, non-iterative filter needs only around $45 \mathrm{~min}$.

In the work presented we describe a method for fast deconvolution of large mosaic image stacks. It combines the experimental determination of aberrations caused by optical components and specimen with a modelled PSF that assumes ideal imaging conditions. We sought to optimize the optical system by minimizing the aberrations induced by its optical components and the cortical tissue - here 100- $\mu$ m-thick cortical slices from rat brains. We experimentally verify that it is possible to obtain nearly ideal imaging conditions. Once these imaging conditions are achieved, the complex process of image formation is simplified by reducing the optical pathway to a single refraction and a single diffraction event. Refraction occurs at the interface between the specimen and the immersion medium, which fills the space between the tissue and the objective lens. Diffraction arises at the finite entrance pupil of this objective lens. We further show that the modelled PSF describes sufficiently accurate the image formation for this combination of specimen and microscope. Therefore a linear, non-iterative deconvolution algorithm already yields significantly improved image quality and resolution.

To verify that the degree of aberrations is indeed negligible and constant for similar specimens (e.g. brain slices from different animals and different cortical depths), we measure aberrations by analysing the deformation of a plane wave front behind the optical system using a Shack-Hartman (SH) wave front sensor (Beverage et al., 2002). The samples were either fluorescent beads in Mowiol or beads between two slices of tissue, also embedded in Mowiol. The resultant measured wave front function is essentially the OTF (Beverage et al., 2002). It yields the amount of aberrations induced by the optical components or the tissue for the two sample types, respectively. This measurement allows then the determination of the average refractive index of the specimen.

The major advantage for using a SH sensor for PSF measurement is that the bead size is not limited by the diffraction limit of the objective (Beverage et al., 2002). Therefore a larger (e.g., 10- $\mu \mathrm{m}$ diameter) and hence brighter bead can be used, which allows the measurement of the PSF over a larger axial range $(10 \mu \mathrm{m})$ even when the bead is embedded within cortical tissue.

Finally we show that the modelled PSF, which is based on measured aberrations that are of a negligible amount, describes the optimized (TLB) imaging system quite well. A high-resolution visualization and quantification of neuronal morphology from linearly deconvolved mosaic data is hence possible.

\section{Materials and methods}

\section{Sample preparation}

The embedding medium used for all presented samples is Mowiol (Carl-Roth-GmbH + Co.KG, 2006). It has a refractive index of $n_{\text {mow }}=1.49$, which is similar to the refractive index of the glass $\left(n_{\text {glass }}=1.516\right)$ that is used for the objective lenses (Olympus, Tokyo, Japan) and the cover slip. Hence, throughout this article oil-immersion objectives are implemented. The immersion medium used is oil (Olympus), having the same refractive index as glass $\left(n_{\text {glass }}=n_{\text {oil }}=1.516\right)$.

To measure the aberrations caused by the optical components, the samples of choice are green fluorescent micro-spheres (beads) with a diameter of $10 \mu \mathrm{m}$ and an emission maximum at a wavelength of $486 \mathrm{~nm}$ (Fluoresbrite Yellow Green; Polysciences Europe $\mathrm{GmbH}$, Eppelheim, Germany). For measuring additional aberrations caused by cortical tissue, we choose fixed 100- $\mu$ m-thick slices prepared from rat cortex, which are usually used for reconstructing the morphology of neurons (Oberlaender et al., 2007). These slices contain biocytin-labelled neuronal processes (dendrites 
and axons) from in vivo filled cortical or thalamic nerve cells. All presented cells are from Wistar rats (P28 - 31; Charles River Laboratory). Acute experiments were carried out in accordance with the animal welfare guidelines of the Max Planck Society.

Neurons were filled with biocytin using either the juxtasomal protocol (Pinault, 1996) or via whole-cell recording (Margrie et al., 2002). The filled neurons were kindly provided by Randy Bruno (Columbia University). Whole-cell recording pipettes were tip-filled with (in $\mathrm{mM}$ ) $135 \mathrm{~K}$-gluconate, 10 Hepes, 10 phosphocreatin-Na, $4 \mathrm{KCl}$, 4 ATP-Mg, 0.3 guanosine triphosphate, and $0.2 \%$ biocytin (pH 7.2, osmolarity 291). Juxtasomal fillings were made similarly with the following differences. Pipettes were filled with CSF containing 1\% biocytin. No pressure was applied to the pipette at any time. Once a cell was isolated by extracellular recordings, current pulses (1-5 nA, 200-ms on, 200-ms off) were applied continuously for several minutes (Pinault, 1996).

The cortex was cut tangentially in 100- $\mu$ m-thick sections. Biocytin in these sections was visualized with the chromogen 3,3'-diaminobenzidine tetrahydrochloride (DAB) using the avidin-biotin-peroxidase method (Horikawa \& Armstrong, 1988). Sections were counterstained for cytochrome oxidase (Horikawa \& Armstrong, 1988). Processed sections were then mounted on slides and cover slipped with Mowiol.

The fluorescent beads were placed between two slices of tissue that were treated as described above. The investigated slices are taken at various cortical depths (i.e. 300-1800 $\mu \mathrm{m}$ from the cortical surface).

\section{Imaging Instrumentation}

A standard brightfield microscope (Olympus BX-51, Olympus, Japan) equipped with a motorized $x y z$-stage (Maerzhaeuser Wetzlar, Germany) was used for image acquisition. It can be used for TLB and fluorescent microscopy. In order to obtain monochromatic illumination, if used in TLB mode, a $546 \pm 5 \mathrm{~nm}$ band pass illumination filter (CHROMA AF-analysentechnik, Germany) was attached to the diaphragm of the lighthouse. The light is then transmitted by a high numerical aperture (1.4NA) oil-immersion condenser (Olympus, Japan). In fluorescent mode, a 100W xenon arc lamp is used with a $470 \pm 10 \mathrm{~nm}$ band pass filter and a $485-\mathrm{nm}$ dichroic mirror (Olympus, U-MGFPHQ) to excite the samples. Fluorescent emission is controlled by the dichroic mirror and a $520 \pm 20 \mathrm{~nm}$ band pass filter.

The specimen is either imaged by a $40 \times$ high numerical aperture oil-immersion objective (Olympus $40 \times$ U PLAN FL $\mathrm{N} ; 1.3 \mathrm{NA}$ ) or a $40 \times$ dry objective (Olympus $40 \times$ U PLAN FL; $0.75 \mathrm{NA}$ ). The stage is navigated in three spatial directions by an OASIS-4i-controller hardware and software (Objective Imaging Ltd, Cambridge, UK). It allows the acquisition of large mosaic images (Chow et al., 2006) at different focal planes. 8-bit grey value images are recorded by a 'Q-icam Fast'
1394 camera (Q-imaging, Canada), equipped with a 4.65- $\mu \mathrm{m}$ CCD pixel element, which yields an $x / y$-sampling of $116 \mathrm{~nm}$ per pixel. The process of mosaic/optical-sectioning imaging is controlled by the Surveyor Software (Objective Imaging Ltd, Cambridge, UK) and has been described in detail previously (Oberlaender et al., 2007).

\section{Shack-Hartmann wave front measurement}

The aberration function $W$, which represents the deviation of the measured wave front from a plane wave, can be expanded as a power series in exit pupil polar coordinates (Beverage et al., 2002; Born \& Wolf, 2003):

$$
W(\rho, \theta)=A_{\ln m} \rho^{n} \cos ^{m} \theta
$$

where $\rho$ represents the normalized pupil coordinate, $\theta$ is the angle between the pupil and the field vectors and $\mathrm{l}, \mathrm{m}, \mathrm{n}$ are integers (Born \& Wolf, 2003). Due to rational symmetry conditions and neglecting of off-axis aberrations, the expansion is limited to $l+n=0,2,4, \ldots$ and $m=0$, respectively. Therefore the aberration function reduces to two terms when expanded to the $4^{\text {th }}$ order and when the $0^{\text {th }}$ order term is ignored, assuming incoherent illumination:

$$
W(\rho)=A_{020} \rho^{2}+A_{040} \rho^{4}
$$

The coefficients $A_{020}$ and $A_{040}$ are expressed in micrometres and represent the wave front deformation caused by defocus and spherical aberration, respectively. These coefficients are measured during the $\mathrm{SH}$ wave front analysis. For a detailed derivation of how the $\mathrm{SH}$ analysis is related to the wave front coefficients see (Beverage et al., 2002).

An illustration of the wave front measurement experiment is shown in Fig. 2. The $\mathrm{SH}$ sensor and the aberration measurement unit are implemented within a 'Wavescope' (Wavescope Model WFS-01, Adaptive Optics Associates, Inc; Cambridge, MA, USA). It comprises a $32 \times 32$ array of square micro-lenses (focal length: $f=3.5 \mathrm{~mm}$, micro-lens spacing: $d=300 \mu \mathrm{m}$ ) to sample the wave front in the exit pupil of the microscope as piece-wise planar wavelets and an optical relay system that guarantees correct illumination of the implemented CCD camera. The 'Wavescope' measures the displacement of spots created by each micro-lens with respect to a prior calibrated reference spot image of a plane wave. It further calculates the coefficients of the aberration function. Coefficients are manually scaled to match the normalization $A_{\ln m}$, as presented above.

To relay the sample image distribution from the microscope's image plane (MIP) on to the micro-lens array of the $\mathrm{SH}$ sensor (P2), a plano-convex singlet (L1) with a focal length of $f_{\mathrm{L} 1}=40 \mathrm{~mm}$ was used and located $40 \mathrm{~mm}$ behind the MIP. Hence, this lens provides the SH sensor with a collimated and de-magnified sample distribution of the exit pupil. A grid of $7 \times 7$ micro-lenses was always illuminated, independent whether the light was supplied by a bead or a laser. This $7 \times$ 7 grid (Fig. 2) was used for the wave front measurements. 
a

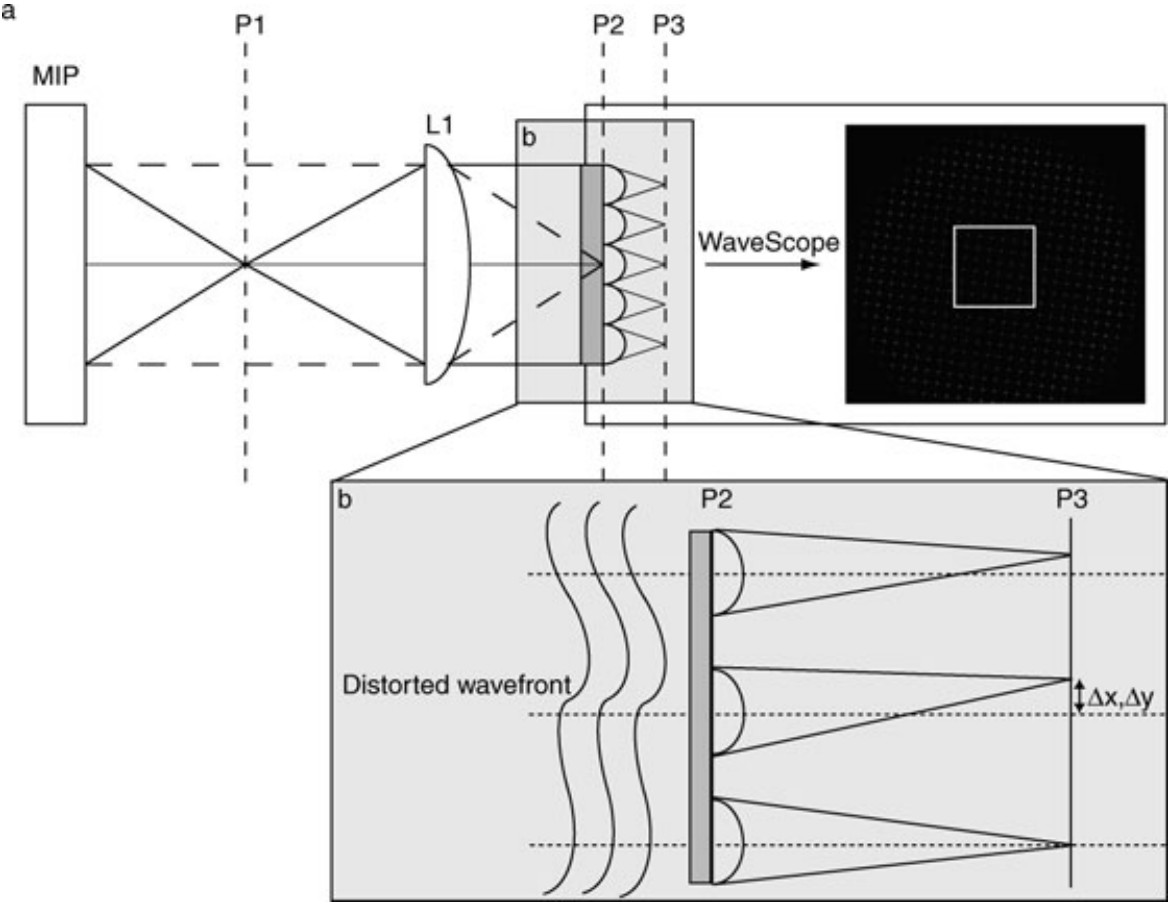

Fig. 2. (a) Illustration of SH wave front analysis. The wave front in the microscope's image plane (MIP) is created by fluorescent beads that are embedded in Mowiol and is relayed by the plano-convex singlet (L1) onto the array of micro-lenses (P2) of the SH sensor. The spot displacements within the according spot image are compared with the spot image of a plane wave created by a laser. The 'WaveScope' measures these spot deviations and relates them to the coefficients of a power series that expresses the aberration function. (b) Principle of the SH wave front sensor as shown in (Beverage et al., 2002). Each lenslet forms an image that is displaced from the local optical axis by an amount $(\Delta x, \Delta y)$. The displacement from the local optical axis is determined by the average slope of the wave front over the lenslet area and the focal length of the lenslet array.

Before recording through-focus SH images of a bead, the SH sensor was calibrated with a monochromatic plane wave. A single mode glass fibre that was connected to a laser (emission wavelength $632.8 \mathrm{~nm}$ ) was therefore located at the MIP, $40 \mathrm{~mm}$ in front of the plano-convex collimator lens (L1). By temporarily placing a shear plate within the light beam between the collimator lens and the lens array, collimated illumination of the $\mathrm{SH}$ sensor could be monitored. The resulting SH reference image defines the imaging properties of the SH sensor, if the exit pupil wave front is described by a plane wave. It accounts for any on-axis aberrations introduced by the sensor optics. Off-axis aberrations can reasonably be neglected due to the size of the micro-lenses (Beverage et al., 2002).

To measure the aberrations caused by the microscope's optical components, a sample with a fluorescent bead embedded in Mowiol was centred in the field of view of the microscope. Through-focus SH images were then recorded at $0.5-\mu \mathrm{m}$ axial intervals over a range of $10 \mu \mathrm{m}$, between the in-focus position of the bead and the cover slip. To improve the SNR, 100 frames were recorded and averaged at each focal position, using an integration time of $60 \mathrm{~ms}$ per frame. For measuring additional optical influences on the light beam caused by the tissue, the same measurement was repeated for a bead sample that was placed between two layers of tissue. These measurements were repeated for eight beads, respectively.

\section{Computing hard- and software}

The deconvolution is carried out using Huygens Professional V2.9 software (Scientific-Volume-Imaging, 1995-2007) running on AMD dual-core 64-bit Opteron servers, equipped with either four CPUs and 32 GB memory (DELTA Computer Products GmbH, Reinbek, Germany) or eight CPUs and 64 GB memory (fms-computer.com, Netphen, Germany). This Huygens deconvolution software is capable of generating a wide-field PSF for ideal imaging conditions and different refractive indices between embedding and immersion medium. It further provides multi-thread implementations of a linear Tikhonov-Miller and an iterative MLE deconvolution filter.

\section{Simplification and modelling of image formation}

As indicated above, the modelling of the image formation within a TLB microscopy is not trivial due to the partially coherent illumination (Born \& Wolf, 2003) of the specimen. 
However, considerations about propagation of mutual coherence within a TLB microscope (Streibl, 1984a, b, 1985) showed that the OTF can be characterized by the ratio of the effective NAs of the condenser and the objective [i.e. ratio between the radii of the entrance pupil of the objective and the illuminating source (microscope field stop)]. It is hence possible to model the OTF for the coherent limit (ratio = 0.1 ), the incoherent limit (ratio $\approx 1$ ) or any intermediate partially coherent case (e.g. ratio $=0.5$ ) (Streibl, 1985). These three settings are illustrated by Fig. $3(\mathrm{a}-\mathrm{c})$ on the example of one $x / y$-plane of a stained neuron. The arrows in Fig. 3(a) and (b) indicate coherency artefacts from out of focus planes. The presented imaging system is always adjusted to meet 'Koehler conditions' (Born \& Wolf, 2003), resulting in an effective numerical aperture (NA) ratio of 1.0 (Fig. 3c). The microscope then approximately satisfies the theoretical incoherence criterion (Streibl, 1985). In consequence, the high contrast images generated by our imaging system are assumed not to suffer from coherency effects. Hence, the TLB imaging system is treated as if incoherent light originates at the specimen, i.e. the image formation is modelled as in the case of a fluorescent microscope.

However, inverting the grey values and hence treating the TLB like a wide-field fluorescent microscope, the question remains of how to determine the optical properties of the imaging components or the tissue, in order to derive the correct PSF of this system. The simplest solution is to assume ideal imaging condition. This means, assuming that neither the optics nor the specimen introduce any aberrations to the path of light. Image formation can then be reduced to a 3D diffraction at the finite entrance pupil of the objective (Philip, 1999; Born \& Wolf, 2003). However, because of the refractive index mismatch between the embedding $\left(n_{\text {mowiol }}=1.49\right)$ and the immersion medium $\left(n_{\text {oil }}=1.516\right)$, this simple model is not sufficient.

Figure 4 shows a geometrical model for this configuration (Visser et al., 1992). Since the cover slip and the objective lens have the same refractive index as the immersion oil, these components can be optically neglected. Image formation can hence be reduced to the refraction at the interface between the embedding and the immersion medium and to the diffraction at the finite entrance pupil of the objective lens. This model is implemented in the Huygens software. The parameters are the two refractive indices, the emission wavelength and the effective NA of the objective.

All of these are known for beads embedded in pure Mowiol. However, the cortical tissue could influence the image formation by causing aberrations due to inhomogeneity of the refractive index. To estimate the possible influences of the tissue we use the following model. We assume that neurons keep their original refractive index, which is approximately $n_{\text {plasma }}=1.35$, and simplify them to spherical cell bodies. The remaining tissue (e.g. extra-cellular space) is assumed to adopt the refractive index of Mowiol. Therefore neurons are
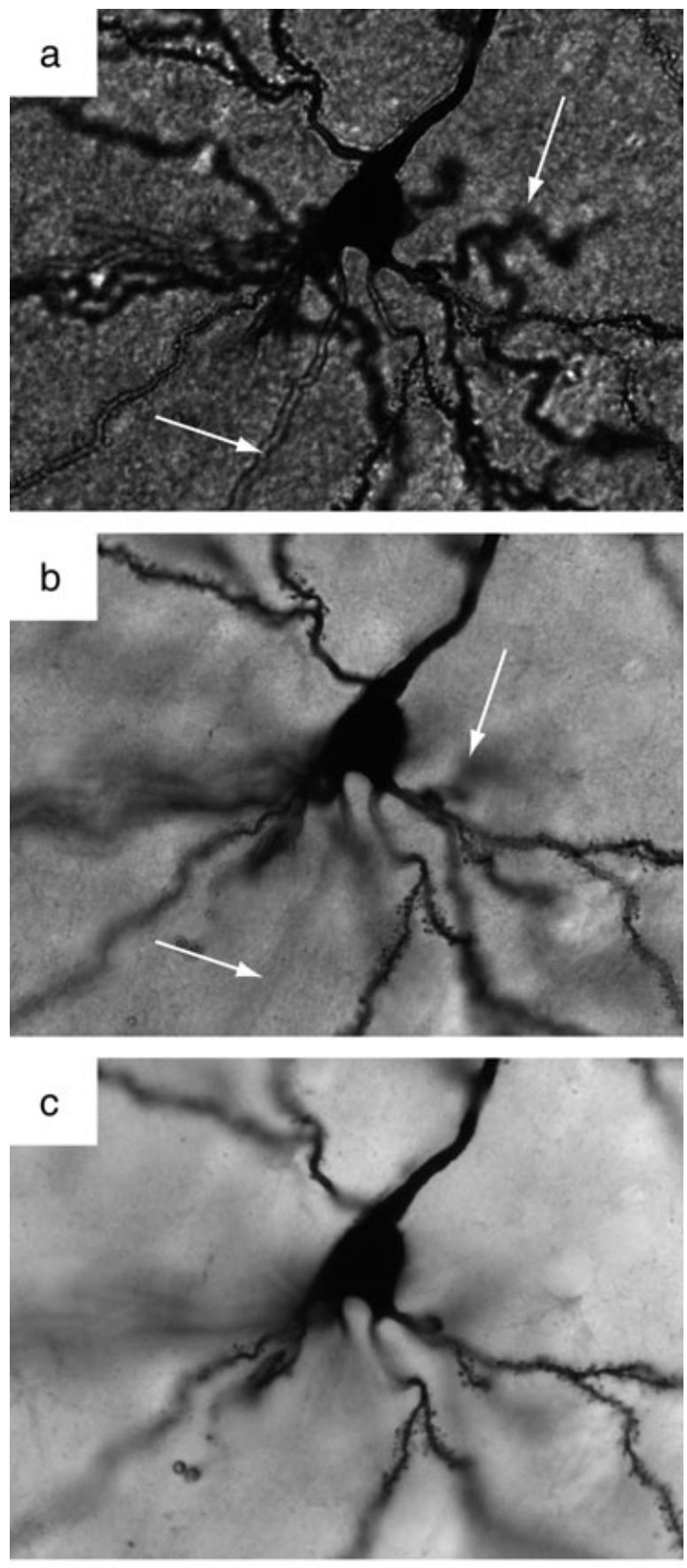

\section{$10 \mu \mathrm{m}$}

Fig. 3. The degree of coherence depends on the ratio of the effective NAs of the imaging system (Streibl, 1985). For illustration an $x / y$-plane of a neuron imaged by the TLB microscope is shown for three different ratio configurations $(0.1 \rightarrow 0.5 \rightarrow 1)$, showing the coherent (a) and incoherent (c) limit, as well as an intermediate case (b). The degree of coherence increases with decreasing ratio. Coherency effects result in out-of-focus artefacts as indicated by the arrows in a and $b$. In the incoherent limit these artefacts are not present (c). This is the configuration for all presented data. It is therefore justified to treat the TLB microscope like a fluorescent one by inverting the grey values. 




Fig. 4. Simple geometrical model (Visser et al., 1992) of the refraction at the interface between a homogeneous specimen $\left(n_{2}\right)$ and an immersion medium $\left(n_{1}\right)$ which fills the space between the specimen and the objective's entrance pupil. It relates optical $(\Delta f)$ and mechanical $(\Delta s)$ defocus and hence allows the derivation of the specimen's refractive index. The angles are slightly exaggerated for illustration.

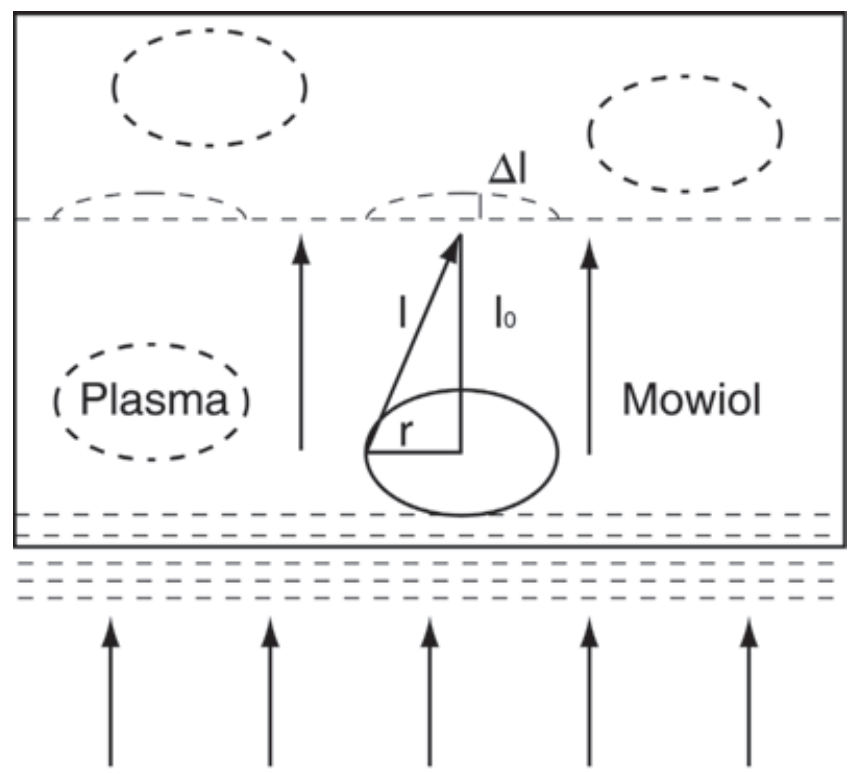

\section{incoherent monochromatic plane wave}

Fig. 5. Simple model illustrating effects of neuronal cell bodies on deformation of a plane wave. If neurons keep their original refractive index, they would function as convex lenses within an environment of Mowiol. This would cause deformations of the wave front and would hence lead to a significant amount of spherical aberrations.

treated like convex lenses with an average radius of $10 \mu \mathrm{m}$ in a homogeneous environment of Mowiol. The focal length of these neuronal lenses is then given by:

$$
f_{\text {cellbody }}=\frac{r}{2} \cdot \frac{1}{n_{\text {mowiol }}-n_{\text {plasma }}}
$$

Therefore, according to Fig. 5 the length difference between a diffracted and a non-diffracted light beam $(\Delta l)$ would be approximately:

$$
l=\sqrt{l_{0}^{2}+r^{2}} \text { and } \Delta l=l-l_{0} .
$$

This simple model yields that the wave front deformation $\Delta l$ is about $1.37 \mu \mathrm{m}$ and therefore already about 2.5 times the wavelength, assuming the light penetrates only one cell body within the image stack.

It is therefore required to show that the cell bodies do not function as spherical lenses and to prove that the influence of the tissue is negligible, by contrast to the above estimation. Otherwise the simple 'refraction plus diffraction' model could not be applied. In the result section this will be described. Further, the average refractive index for the cortical tissue from rat brains is determined. For the subsequent sections on deconvolution this 'refraction plus diffraction' model will always be applied.

\section{Deconvolution algorithms}

Treating the TLB microscope like a fluorescent one and reducing the complex process of image formation to refraction at the interface between embedding and immersion medium and to diffraction at the finite aperture of the objective's entrance pupil, the Huygens software is able to model a PSF. This PSF can then be applied by various algorithms to the inverted mosaic image stacks. We used the linear TM filter and a non-linear, iterative MLE filter. Both of them are already implemented in the Huygens software. However, since Huygens cannot process a 25 GB image stack in one run and estimation of a single background value for such a large volume is rather impracticable, the mosaic image stacks are split up into by 100 pixels overlapping 'bricks' with a lateral size of $3100 \times 3100$ pixels. Thus a background value is estimated for each brick.

By contrast to the MLE filter, the image restoration filter of Tikhonov and Miller has no constraints and yields a result after a single deconvolution step. The MLE algorithm ensures non-negative solutions and needs various iterations before it converges or meets a user defined stop criterion. It is further strongly influenced by the background estimation, making the algorithm ineffective when the background is underestimated or causes a severe performance loss if the background is overestimated. More details about these algorithms and their properties can be found in (van Kempen et al., 1997; van Kempen \& van Vliet, 2000a, b).

\section{Deconvolution samples and resolution measurements}

In order to verify that our assumptions about ideal imaging conditions, incoherent illumination and a homogeneous refractive index of the tissue are correct, we investigated biocytin-labelled neuronal processes (dendrites and axons) within mosaic TLB stacks from the cortex of six different rats and at different cortical depths.

We first checked whether the results from the SH analysis about a homogeneous refractive index of the tissue are confirmed by an alternative approach. This is done by 
comparing the theoretically determined angle of the light cone of the modelled PSF with light cones from 15 dendrites and 15 axons, arbitrarily chosen from various locations and depths within the raw image stacks. These cones were further checked for $z$-dependence and symmetry with respect to the optical axis and the focal plane.

Second, the SNR and the lateral $(x / y)$ as well as the longitudinal $(x / z)$ half-widths are measured from 22 dendrites and 36 axons. This measurement is performed for the raw images and their deconvolved counterparts. These measurements were performed on stacks either containing only dendrites, only axons or both types of structures in intermediate neighbourhood. Since axons are usually fainter and thinner than dendrites, the influence of the background estimation for such 'mixed' stacks will become important. To further illustrate this behaviour, images comprising cylindrical structures that were created in IMAGEJ (Rasband, 2007) are convolved with the modelled PSF. The dimensions are chosen that these structures mimic idealized axons and dendrites that are imaged by a microscope that resembles the properties of the model PSF. Again, stacks containing only idealized dendrites or axons, as well as 'mixed' stacks were deconvolved and the results were compared.

\section{Results}

\section{Aberration measurements for optical components} and cortical tissue

In Fig. 6(a), the measured optical defocus coefficients $\left(A_{020}\right)$ of the aberration function are plotted for a mechanical defocus from 0 to $10 \mu \mathrm{m}$ in $0.5-\mu \mathrm{m}$ steps. Mechanical defocus refers to the physical displacement of the specimen along the optical ( $z-$ ) axis, whereas optical defocus denotes the actual penetration depth into the specimen. If mechanical and optical defocuses are identical, the refraction indices of tissue and immersion media will be identical as well.

Each plot shows measured values averaged from eight samples. Figure 6(a) (plot C) illustrates the $40 \times$ dry objective in combination with fluorescent beads embedded in Mowiol. The optical defocus of almost $15 \mu \mathrm{m}$ for a mechanical defocus of $10 \mu \mathrm{m}$ is caused by the inhomogeneous refractive index within the optical pathway. The refractive index of the air between the specimen and the objective is approximately $1\left(n_{\text {air }}=1.0003\right)$ and therefore refraction effects cause the apparent difference in optical and mechanical defocus. Consequently the use of dry objectives results in a much thinner appearance of the tissue.

The situation is different when the oil-immersion objective is used (Fig. 6a; plot A/B). Figure 6a (plot B) illustrates bead samples embedded in Mowiol. Here the optical defocus is nearly identical to the mechanical one, because the refractive index between the objective and the specimen is homogeneous. The optical path of the light beam is dominated by the refraction

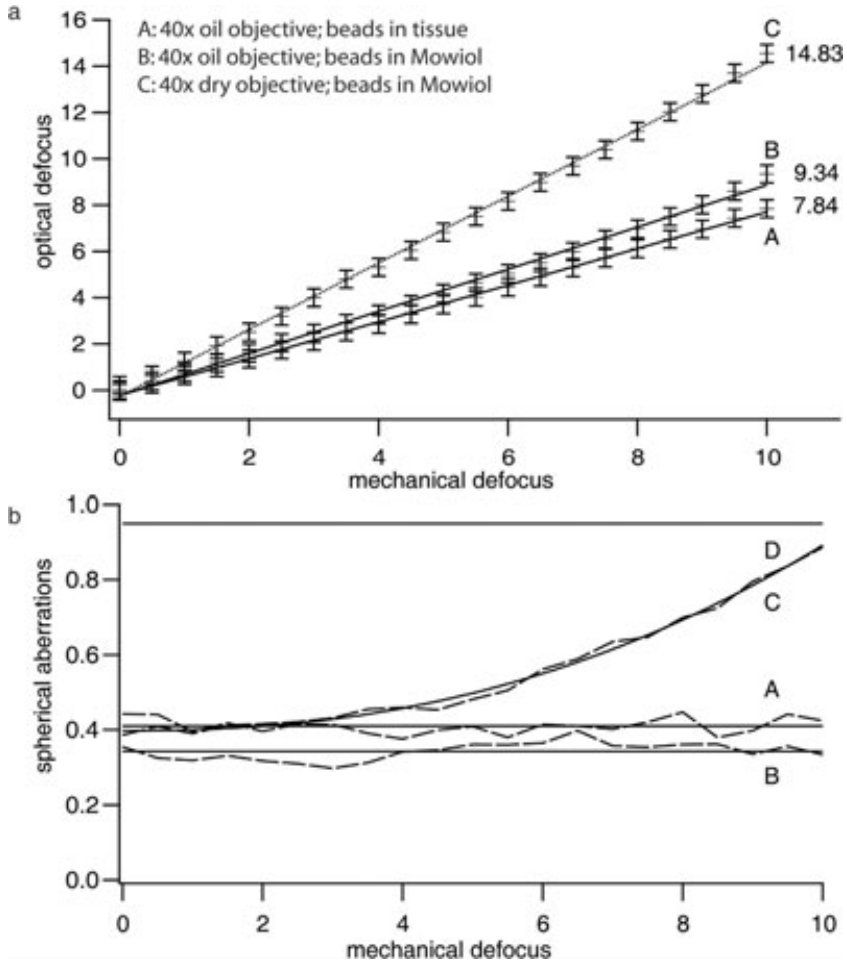

Fig. 6. (a) Measured optical defocus $\left(\mathrm{A}_{020}\right)$ averaged for eight samples each. Whereas beads embedded in Mowiol (B) yield almost identical optical and mechanical defocus, beads between two layers of tissue (A) appear thicker. This suggests a slightly lowered refractive index. (b) Measured spherical aberrations $\left(\mathrm{A}_{040}\right)$ averaged for eight samples each and normalized to the wavelength. As suggested by (a), the spherical aberrations are slightly increased if the beads are embedded in tissue. However, the total amount of aberrations is independent of mechanical defocus for the oil-immersion objective (A/B) and well below one wavelength. By contrast, the spherical aberrations increase rapidly with defocus for the dry objective, reaching the wavelength at about $10 \mu \mathrm{m}$ mechanical defocus.

at the interface between the specimen and the immersion medium. Since the refractive index of the embedding medium is slightly smaller than that of the immersion medium, the specimen appears thicker than it actually is. This is illustrated in a simplified way by the marginal ray model in Fig. 4 (Visser et al., 1992). Based upon this model the optical defocus $\Delta f$ can be related at first order to the mechanical one $\Delta s$ if the numerical aperture $(\mathrm{NA}=1.3)$ and the two refractive indices are known:

$$
\Delta f=\frac{\tan \left(\arcsin \left(\frac{N A}{n_{1}}\right)\right)}{\tan \left(\arcsin \left(\frac{N A}{n_{2}}\right)\right)} \cdot \Delta s .
$$

Using the above formula, the refractive index for bead samples embedded in tissue can be calculated from the measured optical and the known mechanical defocus. At $10 \mu \mathrm{m}$, mechanical defocus the average resulting optical defocus from eight bead samples surrounded by tissue is 
$7.84 \pm 0.55 \mu \mathrm{m}$. Using this value, the approximate refractive index for slices from cortical rat tissue embedded in Mowiol is $n_{\text {tissue }}=1.44 \pm 0.02$. Since the deviation in optical defocus and hence in refractive index is small $( \pm 0.02)$ for bead samples embedded in tissue from different rats and slices, at random positions between these slices, the refractive index is regarded as homogenous for such specimen.

Regarding the tissue as an homogeneous medium with a refractive index of $n_{\text {tissue }}=1.44 \pm 0.02$, the deviations for spherical aberration measured for bead samples between slices of tissue should also be small. This prediction is verified by the results shown in the graphs in Fig. 6(b). Here the average measured coefficient for spherical aberration $A_{040}$ is normalized to the wavelength $\lambda$ of the illuminating light. Both plots for the oil-immersion objective (Fig. 6b; plot A/B) show a constant coefficient for spherical aberration, independent of the mechanical defocus. However, the absolute coefficient value for bead samples between layers of tissue is slightly higher compared to the one for bead samples without tissue. This is reasonable since the major source for this type of aberration is the refractive index mismatch between the embedding and the immersion medium, which is slightly higher for tissue, as shown above.

For completeness the coefficient for spherical aberration for the dry objective was also measured. Figure 6(b) (plot C) shows that the coefficient for spherical aberration increases strongly with increasing defocus reaching the order of the wavelength at $10 \mu \mathrm{m}$ mechanical defocus.

In summary, these measurements suggest that the used oil-immersion objective can be regarded as well corrected with respect to spherical aberration, since the normalized aberration coefficient meets Maréchal's tolerance condition (Born \& Wolf, 2003), which states that aberrations are negligible if the according aberration coefficient $A_{\ln m}$ meets the following equation:

$$
\frac{A_{\ln m}}{\lambda} \leq \frac{3 \sqrt{n+1}}{5 \sqrt{2}}
$$

For spherical aberration $(n=4)$ the normalized coefficient needs to be smaller than 0.94 (Fig. 6b; plot D). Further, the measured spherical aberration is essentially caused by the refractive index mismatch between the embedding and the immersion medium, which is slightly higher if the cortical brain tissue is introduced to the optical pathway. Furthermore, the cortical specimen used can be regarded as homogeneous in refractive index. It is therefore justified to assume ideal imaging condition and to reduce the image formation to the 'refraction plus diffraction' model. Hence, these simplifications of the image formation process are justified for this imaging setup and the modelling of the PSF can be executed. Table 1 shows the used parameters that need to be specified in the Huygens program to calculate a wide-field PSF based upon the above assumptions.
Table 1. Modelling parameters for PSF calculation by Huygens Professional. Ideal imaging conditions are assumed and the optical pathway is reduced to refraction at the interface between embedding and immersion medium and diffraction at the finite entrance pupil of the objective.

\begin{tabular}{ll}
\hline Parameters for PSF modelling in Huygens & Value \\
\hline PSF mode & Widefield \\
Background estimation & Widefield \\
NA & 1.0 \\
$x / y$ sampling & $0.116 \mu \mathrm{m}$ \\
$z$-sampling & $0.5 \mu \mathrm{m}$ \\
Refractive index of immersion medium & 1.516 \\
Refractive index of embedding medium & 1.437 \\
Emission wavelength & $546 \mathrm{~nm}$ \\
Excitation wavelength & $546 \mathrm{~nm}$ \\
\hline
\end{tabular}

\section{Model point spread function and cone of light}

According to the above results of the SH wave front measurements, the tissue is regarded as homogeneous in refractive index $\left(n_{\text {tissue }}=1.44\right)$. Therefore the light sources, in our case the neuronal processes from inverted TLB stacks, should have light cones which resemble the theoretically modelled cone. Figure 7 (a) and (b) shows an $x / z$-plane from an inverted TLB stack with various light cones from neuronal projections and the modelled PSF, respectively. It can be clearly seen that the light cones qualitatively resemble each other. Quantitatively this was shown by measuring four angles, as specified by Fig. 7(c). The mean value for the four angles was between 42.42 and 45.9 degrees $(\alpha 1=44.6 \pm 1.8$; $\alpha 2=42.4 \pm 2.0 ; \alpha 3=43.6 \pm 1.8 ; \alpha 4=45.9 \pm 1.8$ ) (Fig. 8). Hence, the four angles are approximately similar. This is consistent with the assumption of refractive index isotropy. Further, the average angle of a light cone from neuronal projections in cortical tissue embedded in Mowiol, imaged by an oil-immersion objective is $\theta_{2}=44.1 \pm 2.3$ degrees. This value is in good agreement with the theoretical one of $\theta_{2}=44.0 \pm 1.54$ degrees derived from the simple geometrical model, if a NA of 1.0 is assumed for TLB image stacks (Visser et al., 1992). For 'Koehler' illumination the diaphragm of the condenser needs to be set between $2 / 3$ and $4 / 5$ of its maximum size, in our case to a NA of 1.0. This is the NA value for the PSF modelling.

We further checked whether the angles are $z$-dependent. We subdivided the image stacks into four bins with $20-\mu \mathrm{m}$ thickness along the optical axis and calculated the corresponding average angles. Neuronal processes close to the interface between the tissue and the immersion medium $(0-20 \mu \mathrm{m})$ have an average angle of $43.8 \pm 2.1$. The angles for the subsequent bins of $20-40 \mu \mathrm{m}$ and $40-60 \mu \mathrm{m}$ are identical at $44.1 \pm 2.3$. The angles in the most distant region from the cover slip $(60-80 \mu \mathrm{m})$ are $44.9 \pm 2.4$. This implicates a slight 




Fig. 7. (a) $x / z$-plane from an inverted mosaic TLB image stack. The bright structures are characteristic light cones from neuronal projections. Quantitative analysis of these cones from various preparations proved that the cones resemble each other and the cone of the modelled PSF. (b) Central $x / z$-plane of the modelled PSF cone of light. The imaging system is reduced to a simple 'refraction plus diffraction' model and based on the assumptions of ideal imaging conditions and a homogeneous refractive index of 1.44 of the tissue from rat cortex embedded in Mowiol. These assumptions are verified by the SH wave front analysis. (c) Enlargement of two typical light cones from a. All for angles were measured for 15 dendrites and 15 axons from six different rats. The measured average angle is in very good agreement with the theoretically modelled one.



Fig. 8. Illustration of the depth dependence of the tissue's refractive index (i.e. angles of the cones of light). The grey area refers to the expected deviation in angle (i.e. refractive index) according to the $\mathrm{SH}$ analysis $\left(n_{\text {tissue }}=1.44 \pm 0.02\right)$. Angles close to the cover slip are slightly smaller compared to angles at the bottom of the slice. However, the depth dependence is within the error margin of the SH analysis. Hence the refractive index of the tissue is regarded as homogeneous and depth invariant. depth dependence of the refractive index from 1.45 at the top to 1.42 at the bottom of the specimen. However, this deviation is still within the error margin of $1.44 \pm 0.02$ (Fig. 8) that was measured with the SH sensor.

In summary, this angle measurement confirms the $\mathrm{SH}$ analysis by an alternative approach and illustrates the limits of the described PSF simplification. The refractive index of the tissue is not perfectly homogeneous. Both, the $\mathrm{SH}$ analysis, as well as the angle measurement showed small deviations and slight depth dependence of the refractive index. However, for the investigated 100- $\mu \mathrm{m}$-thick slices from cortical tissue and a quantitative tracing of neuronal morphology, these deviations are of a negligible amount.

\section{Deconvolution of cortical samples}

Since the modelled PSF resembles that of the measured one of the imaging system as shown above, a deconvolution should significantly improve the image quality in terms of SNR and resolution. Figure 9 shows the relative loss in signal and background for neuronal processes with inverted grey values from 'bricks' of TLB mosaic image stacks after deconvolution with a linear TM algorithm and an iterative MLE filter, respectively. Whereas the background is reduced by both algorithms in a similar way by approximately $95 \%$, the loss in signal differs significantly. The linear filter reduces the signal amplitude by $42.2 \% \pm 24.4 \%$. By contrast, the MLE filter reduces the signal amplitude by $71.7 \% \pm 17.4 \%$. Consequently the SNR is improved more using the TM filter. 


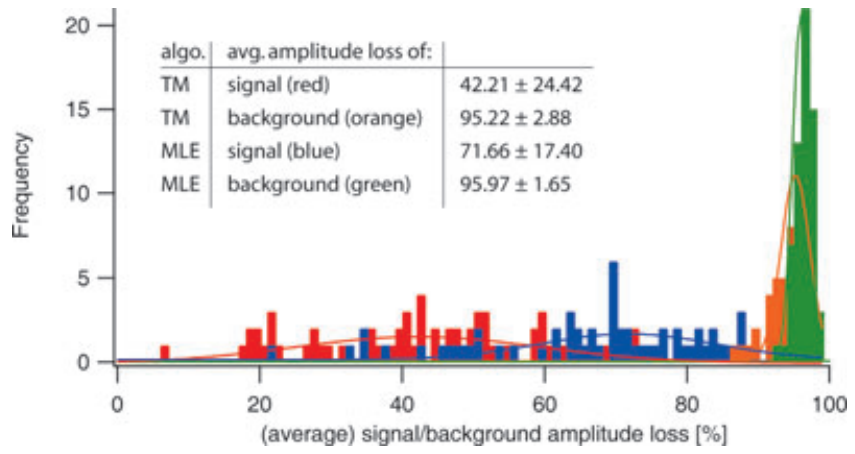

Fig. 9. Illustration of the performance of the deconvolution of inverted mosaic TLB image stacks with the modelled PSF. The loss in signal and background amplitude is shown for a linear TM and an iterative MLE filter. Whereas the background is reduced in a similar fashion by both algorithms, the signal remains stronger after the linear deconvolution. Hence the SNR is more improved by the TM filter.

Table 2. Average ratios of half-width from neuronal projections in raw and deconvolved mosaic/optical-sectioning TLB images. Longitudinal $(z)$ as well as lateral $(x)$ resolution is improved by both deconvolution algorithms. However, the increase in resolution is slightly higher for the linear TM filter.

\begin{tabular}{lll}
\hline & \multicolumn{2}{l}{$\begin{array}{l}\text { Average ratio of half-widths from neuronal projections } \\
\text { in raw and deconvolved image stacks (raw/decon) }\end{array}$} \\
\cline { 2 - 3 } $\begin{array}{l}\text { Deconvolution } \\
\text { algorithm }\end{array}$ & Along optical axis $(z)$ & Along focal plane $(x)$ \\
\hline TM & $1.66 \pm 0.49$ & $1.31 \pm 0.45$ \\
QMLE & $1.53 \pm 0.29$ & $1.20 \pm 0.27$ \\
\hline
\end{tabular}

Table 2 shows the ratios between the half-widths of neuronal processes in raw and deconvolved images, measured along the optical axis (longitudinal; $z$ ) and in the focal plane (lateral; $x$ ). Both algorithms tend to improve the longitudinal resolution more than the lateral one. However, the linear deconvolution performs slightly better (Table 2).

\section{Deconvolution of bead samples}

To further evaluate the goodness of our approach we linearly deconvolved 10 image stacks of beads (10- $\mu \mathrm{m}$ diameter), five embedded in pure Mowiol and five between two layers of tissue at various depths. The deviation from a perfect sphere $(\operatorname{diam}(z) / \operatorname{diam}(x)=1)$ before deconvolution is $0.47 \pm 0.02$ and $0.53 \pm 0.07$, respectively. The slightly thicker appearance along the optical axis of the latter sample type confirms that the cortical tissue decreases the refractive index of Mowiol. After linear deconvolution with the simplified PSF the deviation of the ratio of diameters from unity approaches the spherical limit $(0.09 \pm 0.05$ and $0.12 \pm 0.05)$ (Fig. 10) for both preparations. Due to potential irregularity of the latex beads we conclude that the deconvolution error in object-width is less than $10 \%$.
Further, no significant depth dependence can be found for the beads in tissue. The above measured deviations in refractive index (page 19) of 0.03 between beads close to the cover slip and further away are too small to result in significant diameter changes after deconvolution. This is further illustrated by Fig. 11 where an axonal branch is deconvolved with a PSF modelled with a refractive index for the tissue with 1.44 and 1.49 , respectively. Whereas the half-widths improve by identical amounts, the SNR is slightly higher for the index of 1.44. Assuming ideal imaging conditions, a change in refractive index only changes the angle of the light cone of the PSF from 44.0 to 42.2 degrees. Hence, as long as the refractive index of the tissue is close to the average one of $1.44 \pm 0.02$, the performance of the deconvolution will be similar.

\section{Influence of background estimation}

The estimation of the background value can have a significant influence on the performance of the MLE filter (van Kempen \& van Vliet, 2000b). We therefore checked the loss in signal and background for three groups of structures. First, we investigated dendrites in the absence of the much fainter axons and second, axons in the absence of dendrites. The third group contained both, dendrites and axons. Figure 12 (bold markers refer to mean values) shows the relative signal and background loss for the three groups and for the two algorithms. Whereas the background reduction is essentially independent of the structure type and deconvolution algorithm, the loss in signal amplitude differs significantly (Table 3 ). The tendency that the MLE algorithm decreases the signal amplitude more than the TM filter holds for all three groups. However, whereas the average decrease in signal amplitude is usually much less than the reduction in background, this is not the case for axons surrounded by dendrites. These axons lose around $85 \%$ of their signal amplitude, on average. This means that faint structures, in the presence of much more intense structures, tend to fade after application of a MLE deconvolution filter.

To qualitatively illustrate the effect of the unpredictable performance of the MLE filter for faint structures, we created images containing artificial dendrites (cylinders with a radius of seven pixels in the $x$-direction and three pixels in the $z$-direction; grey value: 254 ) and axons (four pixels in the $x$-direction, three pixels in the $z$-direction; grey value: 133 ). They were convolved with the modelled PSF to mimic imaging by our microscope system. The convolved artificial neuronal projections were then deconvolved by TM and MLE filters, respectively. First only 'dendrites' then only 'axons' and then 'dendrites and axons' were mixed in one stack. In the latter case the TM filter maintains the signal amplitudes in the centre of the structure almost constant, whereas the MLE filter reduces the signal, especially of the 'axons' significantly. It is interesting to note that the TM filter treats identical structures in a similar way, which is not true for the MLE filter. Even 

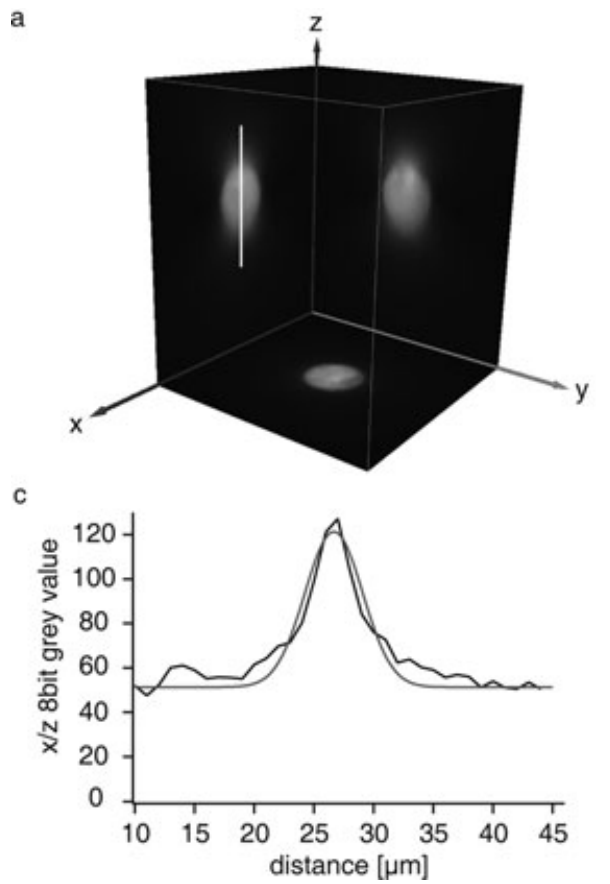

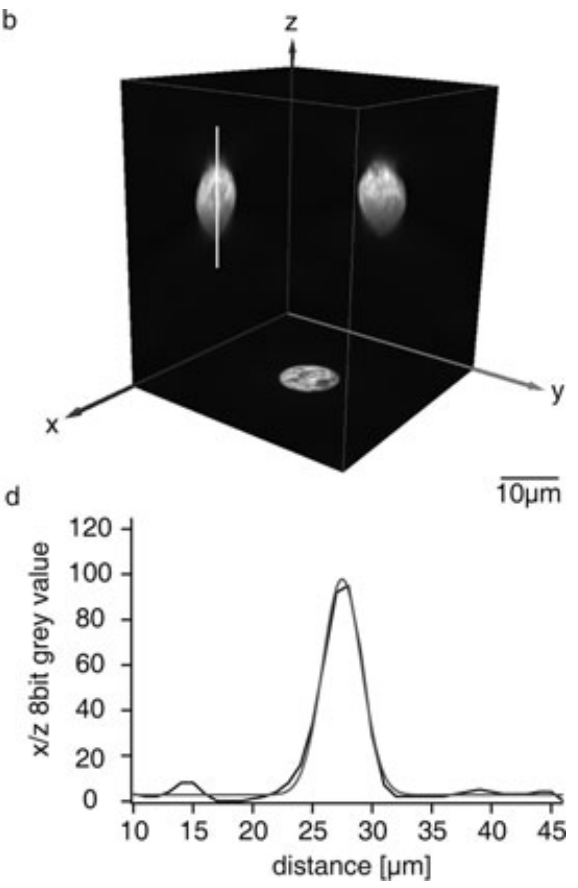

Fig. 10. (a) Projection images along all three spatial planes of a fluorescent bead before deconvolution. (b) Projection images of the same bead after linear deconvolution with the simplified 'refraction plus diffraction' PSF. (c) Z-profile along the white line in (a). (d) Z-profile along the white line in b. Clearly visible is the significant increase in SNR and the decrease in width. The bead approaches the ideal spherical shape after $\operatorname{deconvolution}(\operatorname{diam}(z) / \operatorname{diam}(x)=$ $10.9 / 10=1.09$ ). The same is true for beads between two layers of cortical tissue and no significant depth-dependence of this effect could be found.

though the structures are identical in the original and the convolved image, the two axons differ significantly in their properties after the application of the MLE deconvolution filter (Table 4). This illustrates, that the performance of an iterative deconvolution algorithm on faint structures (axons) can be severely reduced by intense structures (dendrites) located in the intermediate neighbourhood.

Further, the time needed to deconvolve images with a linear filter is much less. Given a stop criterion of $1 \%$ deviation between the results of two iteration steps, the MLE deconvolution needed between 20 and 40 iterations. Hence, the TM algorithm is 20 to 40 times faster and needs less memory, since no intermediate image needs to be stored.

\section{Conclusion and discussion}

We derived an approach for fast deconvolution of large 3D mosaic TLB images of mammalian cortical tissue. Using biocytin-stained neuronal processes from rat cortex as an example we showed that the improvement in SNR and resolution is significant and hence allows quantitative analysis (Oberlaender et al., 2007). This is not possible on the raw images (Holmes \& O'Connor, 2000).

We achieved this by modelling the TLB microscope like a fluorescent one that meets ideal imaging conditions and reduced image formation to refraction at the interface between the embedding and immersion medium and to 3D diffraction at the finite entrance pupil of the objective. These assumptions are verified by measuring the deformation of a plane wave front originating from fluorescent beads embedded in Mowiol or tissue that was soaked with Mowiol with SH wave front analysis. It was shown that spherical aberrations are slightly increased by the tissue, but independent of mechanical defocus or sample position. This result suggests that the tissue can be treated as being homogeneous in refractive index. By measuring the optical defocus and application of a simple geometrical model (Visser et al., 1992) this refractive index was determined to be $1.44 \pm 0.02$.

Similar results were obtained by wave front analysis for twophoton imaging of cortical slices (personal communication, Dr. Markus Rueckel, Max Planck Institute for Medical Research, Germany). In agreement with our results Rueckel finds that the influence of refractive index inhomogeneity within a cortical slice from brain tissue is negligible. Therefore, the gross estimation of aberrations induced by refractive index mismatches between neurons that function as optical lenses proved to be incorrect. Apparently the Mowiol diffuses homogenously into the specimen and results in an average refractive index which is slightly lower than that of Mowiol itself. The assumption that cell bodies function as spherical lenses is hence wrong. In consequence the PSF is independent of neuron density, shape or size. The tissue was taken at various cortical depths from the surface and hence different neuron populations were dominate in each sample [e.g. layer four 

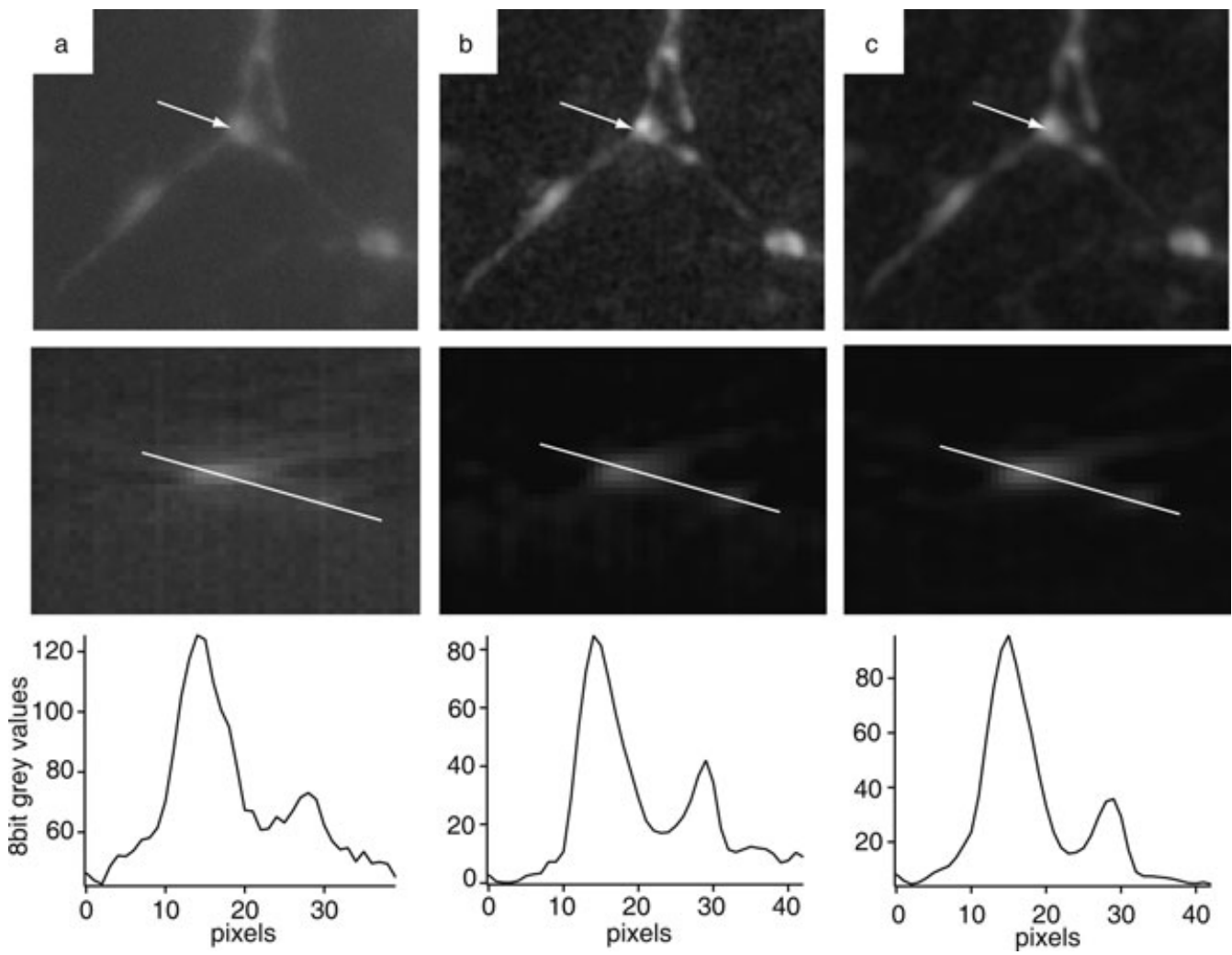

Fig. 11. From top to bottom: (a) Maximum z-projection of an axon within an inverted TLB image stack. The bifurcation is investigated more closely. The figure below shows a $y / z$-reslice of this area. The last figure shows an intensity profile for this bifurcation along the white line. The diverted branch is almost lost within the background noise ( $\mathrm{SNR}=1.5$ ). This makes a quantitative tracing of axonal morphologies impossible. (b) Maximum $z$-projection of the axon shown in figure a after deconvolution with a simplified 'refraction plus diffraction' PSF and an average refractive index of the tissue of 1.44. The downstream figure and the intensity profile illustrate a significant improvement in SNR $=6.4$ and resolution, making a quantitative tracing possible. (c) Same figures as shown in (b). The difference is a refractive index of 1.49 for the PSF modelling. The improvement in resolution and SNR is similar but slightly worse. The effect of a deconvolution with a PSF modelled with a slightly wrong average refractive index is not significant for a quantitative tracing of neuronal morphology.

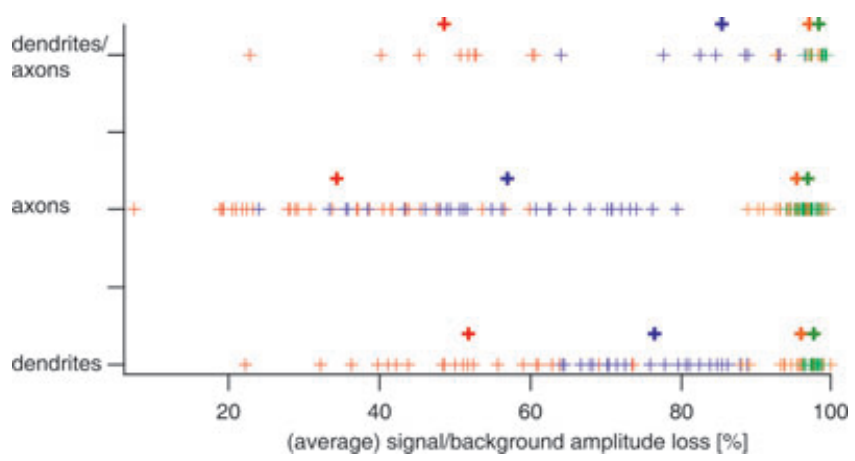

Fig. 12. Illustration of the performance of the two different deconvolution filters for three distinct groups of structure. Whereas the TM (signal/background reduction $=\mathrm{red} /$ orange) filter treats stacks containing only dendrites, axons or axons in the presence of dendrites in a similar way, the MLE (blue/green) algorithm tends to fade away the fainter axonal structures in the presence of more intense dendrites.
Table 3. Average loss of signal and background amplitude for neuronal projections. Whereas the SNR for all three kinds of image stacks is significantly improved by the TM filter, the MLE filter tends to fade fainter structures (axons) in the presence of more intense ones (dendrites).

\begin{tabular}{|c|c|c|c|c|}
\hline \multirow[b]{3}{*}{ Type of image stack } & \multicolumn{4}{|c|}{ Average amplitude loss [\%] } \\
\hline & \multicolumn{2}{|c|}{ TM deconvolved } & \multicolumn{2}{|c|}{ MLE deconvolved } \\
\hline & Signal & Background & Signal & Background \\
\hline Only dendrites & 51.76 & 95.86 & 76.41 & 97.54 \\
\hline Only axons & 34.33 & 95.25 & 56.93 & 96.71 \\
\hline $\begin{array}{l}\text { Axons in the presence } \\
\text { of dendrites }\end{array}$ & 48.54 & 96.91 & 85.33 & 98.20 \\
\hline
\end{tabular}

spiny stellates or layer five pyramidal neurons (Schubert et al., 2003)]. However, the SH measurements were independent of the sample (i.e. independent of the dominant neuron population), confirming the assumption that Mowiol diffuses homogeneously into the tissue. 
Table 4. Signal amplitudes for idealized neuronal projections. Whereas the linear algorithm treats all structures in a similar way, the MLE filter induces unpredictable deviations, especially of 'axons' with 'dendrites' in their immediate neighbourhood.

\begin{tabular}{lllll}
\hline & \multicolumn{4}{l}{ Signal amplitude at the centre of the structure } \\
\cline { 2 - 5 } Idealized structure type & Raw & Convolved & TM deconvolved & MLE deconvolved \\
\hline Dendrite 1 (only dendrites) & 86 & 252 & 250 & 199 \\
Dendrite 2 (only dendrites) & 86 & 254 & 254 & 180 \\
Dendrite 1 (dendrites in the presence of axons) & 86 & 252 & 250 & 98 \\
Dendrite 2 (dendrites in the presence of axons) & 86 & 254 & 254 & 104 \\
Axon 1 (only axons) & 86 & 253 & 250 & 88 \\
Axon 2 (only axons) & 69 & 252 & 248 & 49 \\
Axon 1 (axons in the presence of dendrites) & 69 & 133 & 137 & 106 \\
Axon 2 (axons in the presence of dendrites) & 69 & 133 & 136 & \\
\hline
\end{tabular}

By application of the modelled PSF by a linear TM and an iterative MLE deconvolution filter, it could be shown that the TM filter is superior to the MLE filter in improving the SNR and in robustness. With robustness we refer to the influence of the background estimation for iterative filters (van Kempen \& van Vliet, 2000a, b). Since we are imaging large areas of tissue with a TLB microscope one can assume that the background deviates within such a volume. In areas containing many intense structures, the background will be estimated higher than in regions were only faint structures are present. Hence an overestimation of the background in areas where intense and faint structures are in intermediate neighbourhood leads to fading of the weaker structures when the iterative MLE algorithm is applied. Therefore, the deconvolution of 3D mosaic TLB images from large areas of tissue by an iterative MLE filter will always be problematic and cannot be generalized if intense and faint structures are within immediate neighbourhood. This problem does not arise if a linear filter is applied and prohibits a general application of the iteration based 'blind deconvolution' method.

In conclusion we suggest that mosaic/optical-sectioning TLB microscopy in combination with a linear deconvolution, which is based on experimentally verified assumptions about an ideal imaging system, is a competitive alterative to standard fluorescent techniques for imaging of large volumes of cortical tissue with sub-micrometre resolution. Even without measuring the wave front by $\mathrm{SH}$ analysis, one can still apply a deconvolution with reasonable parameters. By measuring the angles of the light cones for representative structures from different preparations, the above assumptions could be determined indirectly. If the deviations and $z$-dependence of the angles are small, an average angle can be determined. This would further yield the approximate average refractive index of the tissue. However, if the refractive index of the tissue deviates strongly from the one of the immersion medium, resulting in an amount of spherical aberration that is no longer negligible, the application of a PSF for an ideal imaging system is impossible. Hence, only if a combination of embedding and immersion medium can be found that nearly homogenizes the optical pathway (i.e. if the resulting refractive index of the tissue is similar to the refractive index of the immersion medium), this method can be applied for other samples of absorbing structures from biological tissue.

\section{Acknowledgements}

We thank Dr. Randy Bruno for providing cortical tissue containing stained neurons. Special thanks to Dr. Guenther Giese and Dr. Hans van der Voort for fruitful discussions about image formation and microscopy techniques and to the Max Planck Institute for Astronomy for allocation of the 'Adaptive Optics Laboratory'.

\section{References}

Agard, D.A. (1984) Optical sectioning microscopy: cellular architecture in three dimensions. Annu. Rev. Biophys. Bioeng. 13, 191-219.

Agard, D.A., Hiraoka, Y., Shaw, P. \& Sedat, J.W. (1989) Fluorescence microscopy in 3 dimensions. Meth. Cell Biol. 30, 353-377.

Beverage, J.L., Shack, R.V. \& Descour, M.R. (2002) Measurement of the three-dimensional microscope point spread function using a ShackHartmann wavefront sensor. J. Microsc. 205, 61-75.

Born, M. \& Wolf, E. (2003) Principles of Optics. Cambridge University Press, Cambridge, UK.

Bronshtein, I.N., Semendyayev, K.A., Musiol, G. \& Muehlig, H. (2003a) Handbook of Mathematics, p. 633. Spinger-Verlag.

Bronshtein, I.N., Semendyayev, K.A., Musiol, G. \& Muehlig, H. (2003b) Handbook of Mathematics, p. 711. Spinger-Verlag, Berlin, Heidelberg, Germany.

Carl-Roth-GmbH + Co.KG. (2006) Instructions for use Mowiol. 488. www.carl-roth.de/media/-de-de/usage/0793.pdf. Last accessed: 03/01/2008.

Chomik, A., Dieterlen, A., Xu, C., Haeberle, O., Meyer, J.J. \& Jacquey, S. (1997) Quantification in optical sectioning microscopy: a comparison of some deconvolution algorithms in view of 3D image segmentation. J. Optics-Nouv. Rev. D Optique 28, 225-233.

Chow, S.K., Hakozaki, H., Price, D.L. et al. (2006) Automated microscopy system for mosaic acquisition and processing. J. Microsc. 222, 76-84. 
Dodt, H.U., Leischner, U., Schierloh, A. et al. (2007) Ultramicroscopy: three-dimensional visualization of neuronal networks in the whole mouse brain. Nat. Meth. 4, 331-336.

Dusch, E., Dorval, T., Vincent, N., Wachsmuth, M. \& Genovesio, A. (2007) Three-dimensional point spread function model for line-scanning confocal microscope with high-aperture objective. J.Microsc. 228, 132138.

Frieden, B.R. (1967) Optical transfer of 3-dimensional object. J. Opt. Soc. Am. 57, 56-66.

Gibson, S.F. \& Lanni, F. (1991) Experimental test of an analytical model of aberration in an oil-immersion objective lens used in 3-dimensional light-microscopy. J. Opt. Soc. Am. A Opt. Image Sci. Vis. 8, 1601-1613.

Hiraoka, Y., Sedat, J.W. \& Agard, D.A. (1990) Determination of 3-dimensional imaging properties of a light-microscope system-partial confocal behavior in epifluorescence microscopy. Biophys. J. 57, 325333.

Holmes, T.J. \& O'Connor, N.J. (2000) Blind deconvolution of 3D transmitted light brightfield micrographs. J. Microsc. 200, 114-127.

Horikawa, K.\& Armstrong, W.E. (1988) A versatile means of intracellular labeling: injection of biocytin and its detection with avidin conjugates. J. Neurosci. Meth. 25, 1-11.

Lee, S., Kang, M. \& Park, K. (1998) CCD Noise filtering based on 3-dimensional nonlinear partial differential equation. IEEE Trans. Consum. Electron. 44, 1086-1090.

Margrie, T.W., Brecht, M. \& Sakmann, B. (2002) In vivo, low-resistance, whole-cell recordings from neurons in the anaesthetized and awake mammalian brain. Pflugers Arch. 444, 491-498.

Oberlaender, M., Bruno, R.M., B., S. \& Broser, P.J. (2007) Transmitted light brightfield mosaic microscopy for 3D tracing of single neuron morphology. J. Biomed. Opt. 12(6), 064029-1-064029-19.

Philip, J. (1999) Optical transfer function in three dimensions for a large numerical aperture. J. Mod. Opt. 46, 1031-1042.

Pinault, D. (1996) A novel single-cell staining procedure performed in vivo under electrophysiological control: morpho-functional features of juxtacellularly labeled thalamic cells and other central neurons with biocytin or neurobiotin. J. Neurosci. Meth. 65, 113-136.
Rasband, W. (2007) ImageJ 1.38x. http://rsb.info.nih.gov/ij/; last accessed date: 03/01/2009.

Schmitt, S., Evers, J.F., Duch, C., Scholz, M. \& Obermayer, K. (2004) New methods for the computer-assisted 3-D reconstruction of neurons from confocal image stacks. Neuroimage 23, 1283-1298.

Schubert, D., Kotter, R., Zilles, K., Luhmann, H.J. \& Staiger, J.F. (2003) Cell type-specific circuits of cortical layer IV spiny neurons. J. Neurosci. 23, 2961-2970.

Scientific-Volume-Imaging. (2007) Huygens Professional 2.9. http:// www.svi.nl; last accessed date: 03/01/2009.

Streibl, N. (1984a) Depth transfer by an imaging-system. Optica. Acta. 31, 1233-1241.

Streibl, N. (1984b) Fundamental restrictions for 3-d light distributions. Optik 66, 341-354.

Streibl, N. (1985) 3-Dimensional imaging by a microscope. J. Opt. Soc. Am. A Opt. Image Sci. Vis. 2, 121-127.

van Kempen, G.M. \& van Vliet, L.J. (2000a) Background estimation in nonlinear image restoration. J. Opt. Soc. Am. A Opt. Image Sci. Vis. 17, 425-433.

van Kempen, G.M. \& van Vliet, L.J. (2000b) The influence of the regularization parameter and the first estimate on the performance of tikhonov regularized non-linear image restoration algorithms. J. Microsc. 198 (Pt 1), 63-75.

van Kempen, G.M., van Vliet, L.J. \& Vandervoort, H.T.M. (1997) A quantitative comparison of image restoration methods for confocal microscopy. J. Microsc. 185, 354-365.

Vandervoort, H.T.M. \& Strasters, K.C. (1995) Restoration of confocal images for quantitative image-analysis. J. Microsc. 178, 165181.

Visser, T.D., Oud, J.L. \& Brakenhoff, G.J. (1992) Refractive-index and axial distance measurements in 3-d microscopy. Optik 90, 1719.

Wearne, S.L., Rodriguez, A., Ehlenberger, D.B., Rocher, A.B., Henderson, S.C. \& Hof, P.R. (2005) New techniques for imaging, digitization and analysis of three-dimensional neural morphology on multiple scales. Neurosci. 136, 661-680. 